Supporting Information for

\title{
Efficient frequency mixing of guided surface waves by atomically
} thin nonlinear crystals

\author{
Quanbing Guo, ${ }^{1}$ Zhenwei Ou, ${ }^{1}$ Jibo Tang, ${ }^{2}$ Jing Zhang, ${ }^{1}$ Fengya Lu, ${ }^{3} \mathrm{Ke} \mathrm{Wu},{ }^{1}$ Douguo
}

Zhang, ${ }^{3}$ Shunping Zhang, ${ }^{1, *}$ and Hongxing $\mathrm{Xu}^{1,2, *}$

${ }^{1}$ School of Physics and Technology, Center for Nanoscience and Nanotechnology, and Key Laboratory of Artificial Micro- and Nano-structures of Ministry of Education, ${ }^{2}$ The Institute for Advanced Studies, Wuhan University, Wuhan 430072, China ${ }^{3}$ Institute of Photonics, Department of Optics and Optical Engineering, University of Science and Technology of China, Hefei, Anhui 230026, China

\section{Materials and Methods}

\section{Sample fabrication}

The multilayers substrates were fabricated by PECVD (Oxford System 100) on a pre-clean cover glass at $0.1 \mathrm{mTorr}$ and $300{ }^{\circ} \mathrm{C}$. The $160 \mathrm{~nm} \mathrm{SiO}$ and $110 \mathrm{~nm} \mathrm{Si} 3 \mathrm{~N}_{4}$ were periodically deposited and the thickness of the top $\mathrm{SiO}_{2}$ is $340 \mathrm{~nm}$. There are 8 pairs of dielectric materials. To efficiently launch the BSWs, a pair of grating couplers were fabricated on the dielectric multilayers via electron-beam lithography (Raith eLINE Plus) followed by a reactive ion etching. A monolayer $\mathrm{WS}_{2}$ was firstly mechanically exfoliated on a clear Si substrate with $300 \mathrm{~nm}$ thermally grown $\mathrm{SiO}_{2}$, and identified by the bright-field image contrast under a microscope. Then the monolayer $\mathrm{WS}_{2}$ with a proper shape was transferred on the multilayer substrate via the dry transfer method. The multilayer PC with $\mathrm{WS}_{2}$ was annealed to remove the absorbed impurities on the surface of $\mathrm{WS}_{2}$ and ensured tightly adhesive.

\section{Optical measurements}

The femtosecond pulsed laser (LEO, Daheng Optics) with wavelength centered at $800 \mathrm{~nm}$ (pulse duration $46 \mathrm{fs}$, repetition rate $80 \mathrm{MHz}$ ) was separated into two arms and simultaneously launched the BSWs through an infinite-corrected objective $(50 \times$, $\mathrm{NA}=0.5$, Olympus) in the nonlinear optical measurement. The excitation wavelength was selected by a tunable bandpass filter (12-nm bandwidth, Semrock) in the SFG 
33 measurement. The SHG and SFG distribution in the real or Fourier space were 34 imaged by two CCDs. The nonlinear signals were reflected into a spectrometer 35 (Horiba 320, grating 150 lines $/ \mathrm{mm}$ ) equipped with an air-cooled CCD for spectra 36 measurements. In the polarization-resolved SHG measurements, the sample was 37 mounted on a rotatable stage. The picosecond laser $(800 \mathrm{~nm})$ was used to illuminate 38 the $\mathrm{WS}_{2}$ and a co-linear configuration of the excitation and collection polarization was 39 employed. The spectra were obtained by another spectrometer (Horiba 550, grating 40150 lines $/ \mathrm{mm}$ ). For the typical photoluminescence and Raman characterization, a 41 continue-wave $532 \mathrm{~nm}$ laser was focused on the sample through a $100 \times$ objective (NA $42=0.9$, Olympus). The signals were guided through the same objective into a 43 spectrometer (Renishaw, invia) and analyzed by an equipped CCD.

\section{Waveguide mode analysis}

45 All the analyses about the waveguide mode were carried out using the commercial 46 software COMSOL Multiphysics 5.2a. A two-dimensional model was established and 47 the periodic boundary condition was used to simulate an infinite surface. The 48 simulated geometry parameters were taken from the experiment, as described above. 49 The refractive indexes of $\mathrm{SiO}_{2}$ and $\mathrm{Si}_{3} \mathrm{~N}_{4}$ were set as $1.47+10^{-5} \mathrm{i}$ and 2.073 , which 50 were measured using the commercial ellipsometer and can be adjusted by controlling 51 the fabrication conditions. The refractive index of glass was 1.515. The TE-polarized 52 field distribution of BSW mode at a certain frequency was obtained by mode analysis. 


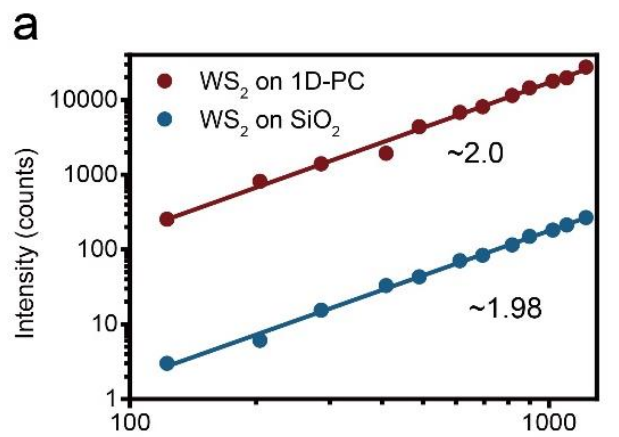
wavelengths. nonlinearity ${ }^{1}$. b

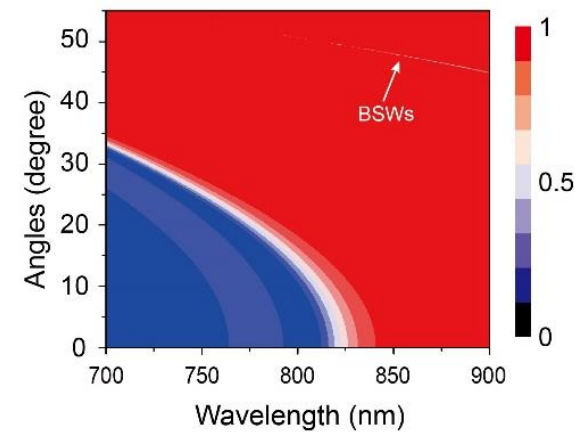

Figure S1. (a) In-situ excited SHG dependent on the incident power for $\mathrm{WS}_{2}$ on the silica and 1D-PC. (b) The calculated reflection efficiency of the dielectric PC near the FW

Dielectric PC can be used to enhance the light-matter interaction due to the increased reflection of light in the optical bandgap region. Figure S1a shows the SHG power dependence under a normal illumination when the $\mathrm{WS}_{2}$ was on different substrates. The quadratic increase of SHG as the laser power indicates the second-order nonlinear process. However, the SHG intensity for $\mathrm{WS}_{2}$ on the dielectric PC was enhanced by two orders of magnitude compared with that on the silica substrate. Simulations were performed to address this enhanced nonlinearity. Figure S1b shows the calculated reflection spectra around the FW wavelength for the bare dielectric PC excited from the substrate. The wavelength range with large reflection efficiency is corresponding to the bandgap in the dielectric multilayer. The BSW modes ranging from 730 to $910 \mathrm{~nm}$ appear as a narrow low-reflectivity region within the photonic bandgap. The enhanced reflection of FW light provides an excitation enhancement at $800 \mathrm{~nm}$, which accounts for the enhanced

\section{S2 Electric field enhancement of BSWs and optimization of the structure}

To address the electric field enhancement of BSWs, an incident plane wave was used to excite the surface mode in a Kretschmann configuration in the simulation. The incident angle is set as $49.69^{\circ}$, which resonantly excite the BSWs at $800 \mathrm{~nm}$ as shown in Fig. S2a. To further determine the enhancement factor of BSWs, the cross-section profile of the BSWs electric field was extracted. Figure S2b shows the calculated field intensity enhancement for the $800 \mathrm{~nm}$ BSWs, which is normalized to the incident wave. Near to the top surface, the electric field intensity displays a maximum enhancement of 50 folds. 
To further improve the spatial overlap of BSWs and $\mathrm{WS}_{2}$, the thickness of the top $\mathrm{SiO}_{2}$
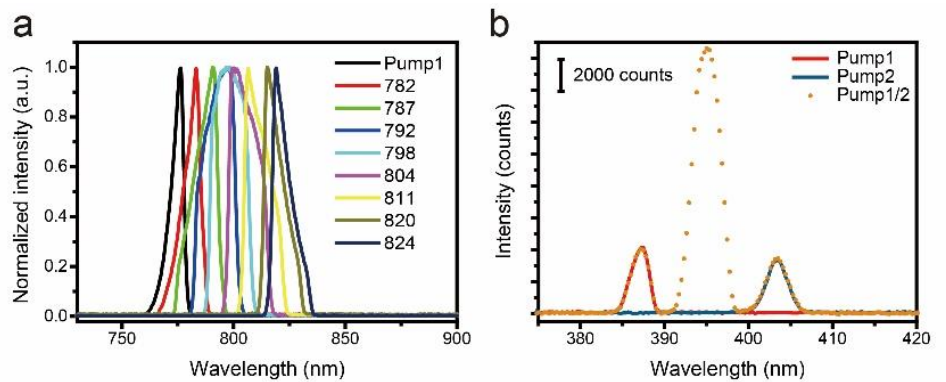
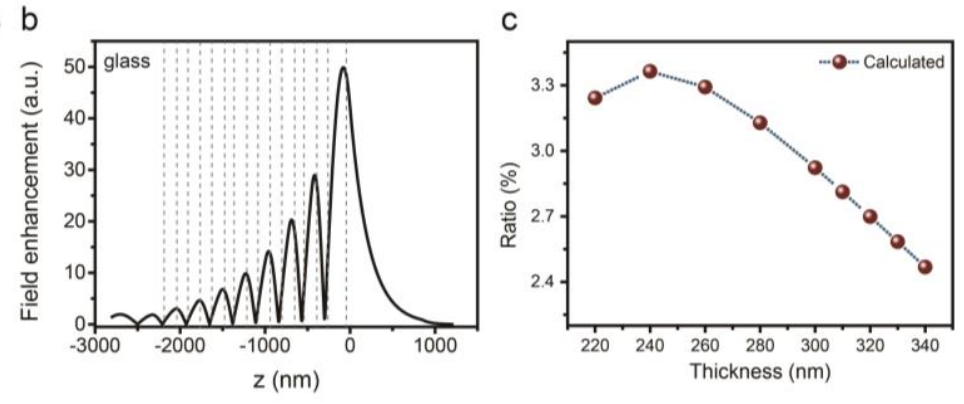

Figure S2. (a) Calculated electric field intensity distribution of BSW mode at $800 \mathrm{~nm}$. (b) FieldintensityenhancementofBSW snormalized totheincidentplanewave.(c)Theenergy density ratio for the BSWs squeezed in the $\mathrm{WS}_{2}$ layer as a function of the thickness of the top $\mathrm{SiO}_{2}$.

\section{S3 Selective FW and corresponding SFG spectra}

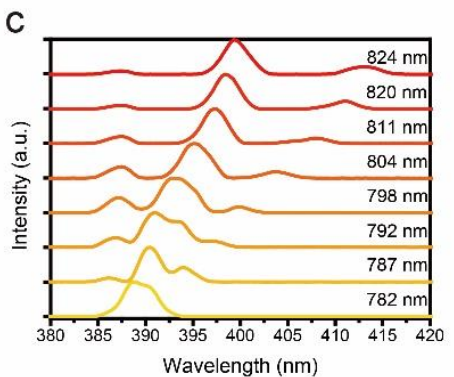

Figure S3. (a) FW spectra selected by the tunable bandpass filter.(b) In-situ SHG and SFG spectra for $\mathrm{WS}_{2}$ on the dielectric multilayer. (c) The normalized SFG spectra evolution when fixing one pump and sweeping the wavelengths of the other pump.

Limited to theavailable spectrum rangeof thelaser source, atunablebandpass filter(12-nm bandwidth, Semrock) was utilized to select the desired pump2 energies in our experiment. Rotating the angles of the filter, the selected FW spectra were shown in Fig. S3a. When the $\mathrm{WS}_{2}$ was pumped by a single beam, only one SHG peak appeared in the high-frequency region. However, for the case two pump beams simultaneously excited the $\mathrm{WS}_{2}$, an additional peak corresponding to the SFG emerged. The comparison spectra were shown 
in Fig. S3b. Fixing pump1 and sweeping the pump2 wavelength from 782 to $824 \mathrm{~nm}$, a full set of nonlinear spectra were recorded and shown in Fig. S3c. The SFG peaks shift as the pump2 wavelength and the emission intensity is larger in comparison with the SHG. We note that the SFG spectra exhibit an asymmetric distribution and increased intensity with the pump2 wavelength, which are attributed to the irregular excitation shapes and the increasing collection efficiency of the setup at a longer wavelength, respectively.

a

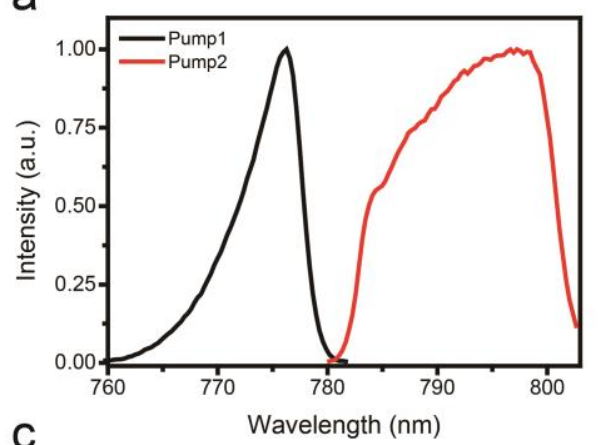

C

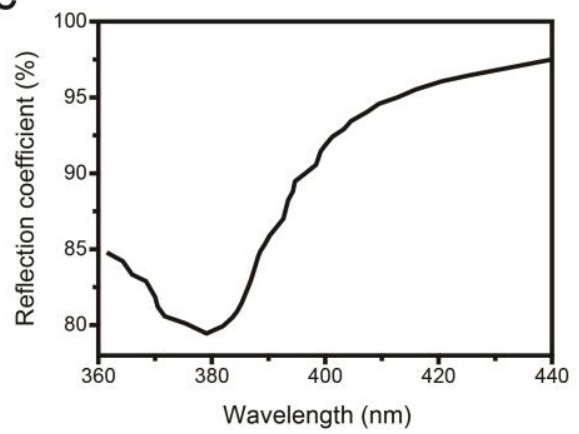

b

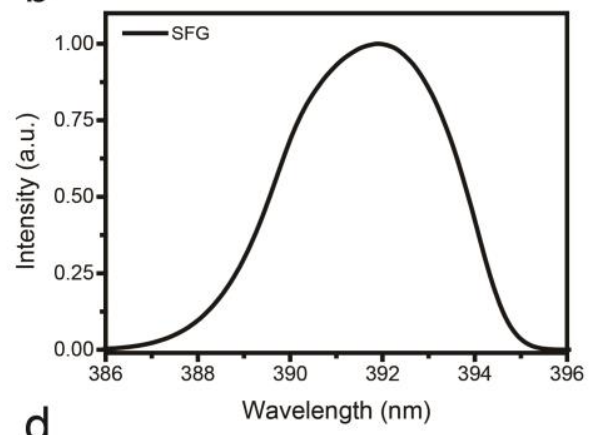

d

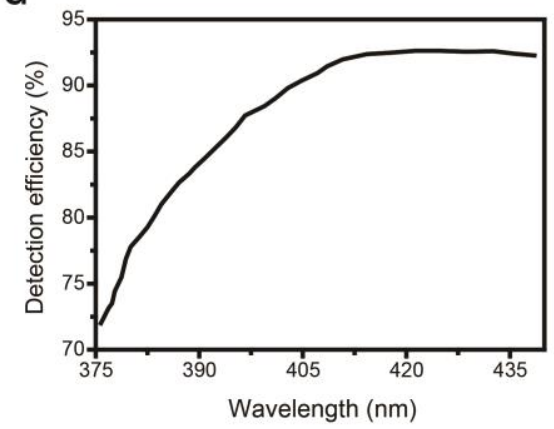

112 Figure S4 (a) The selected spectrum of the pump1 and pump2 (792 nm). (b)

113 Generated sum-frequency spectrum by pump1 and pump2. (c) The reflection coefficient of the mirrors around the SFG frequency. (d) The detection efficiency of the spectrograph as a function of the wavelength.

We note that there appear two peaks corresponding to the SFG for the pump1 and pump2 at $792 \mathrm{~nm}$, which is attributed to the irregular spectra shapes of the pump excitation. Due to the spectral availability of our laser, we use a bandpass filter (bandwidth $12 \mathrm{~nm}$ ) to select the excitation wavelength. The selected spectra were not Gaussian shapes but an irregular distribution depending on the wavelengths, as shown in Fig. S4a. With the pump1 spectrum fixed, we used every frequency component in the pump2 to generate SF spectra by mixing with pump1 and superposed all the spectra. The calculated SFG spectrum is shown in Fig. S4b. Apparently, there are two

125 peaks which account for the obtained SFG spectra in the experiment. Additionally, the SHG intensity of the pump 2 increases with the excitation wavelength, which is 
127 mainly due to the improvement of the reflection efficiency of the silver-coated mirrors 128 (Fig. S4c) and the detection efficiency of the CCD (Fig. S4d). Therefore the SHG 129 intensity accordingly increased, as well as the SFG intensity.

\section{S4 Experimental setup}

131

132 Figure S5. The schematic drawing of the optical measurement setup.

133

134 A femtosecond laser was used to launch the BSWs via the gratings couplers. As shown 135 in Fig. S5, the laser was split into two beams by a beam-splitter. In one beam path, a delay 136 line was used to tune the relative time delay between the two beams. Two bandpass filters 137 were inserted in each path to select pump beams with different energy, which then were 138 focused on the gratings by a $50 \times$ objective ( $\mathrm{NA}=0.5$, Olympus). In the collection path, 139 the FW was blocked by $90-\mathrm{nm}$ bandpass filters (center wavelength at $400 \mathrm{~nm}$, Semrock). 140 The passed SFG light was recorded by a CCD for real-space imaging or back-focal plane 141 imaging. For the spectra measurement, the signals were guided into a spectrometer for the 142 spectra analysis. 
a

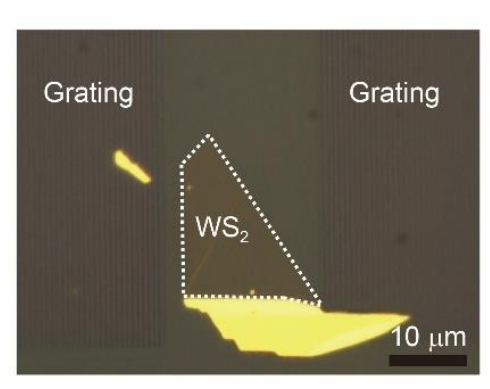

C

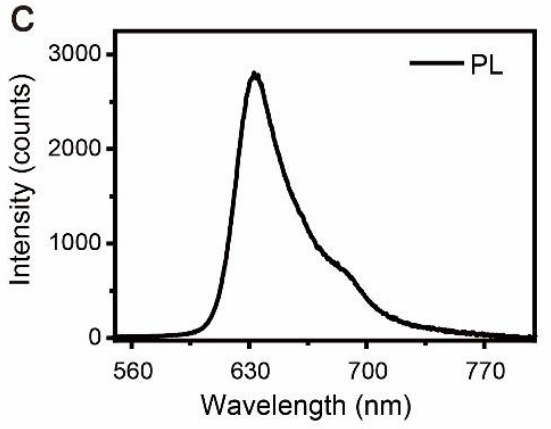

b

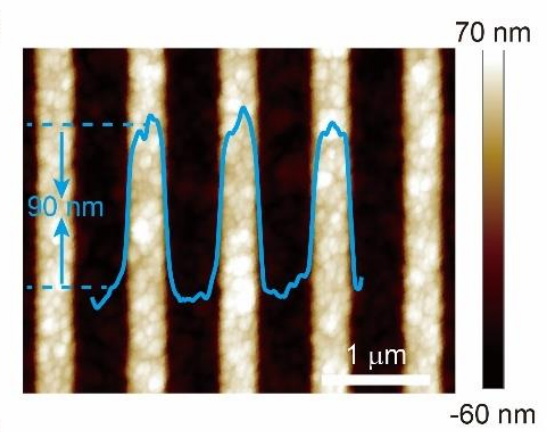

d

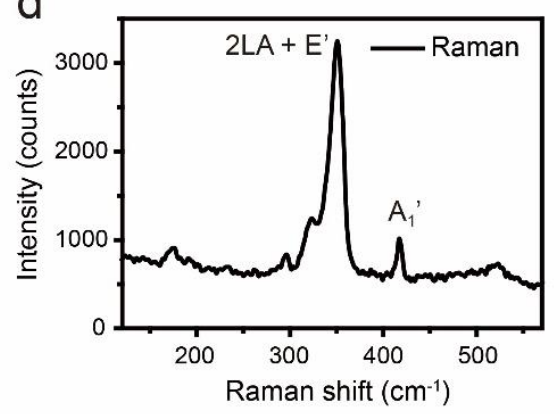

144

Figure S6. (a) Microscopic optical image for $\mathrm{WS}_{2}$ on the dielectric multilayer. The white dashed line marks the monolayer $\mathrm{WS}_{2}$ region. (b) Atomic force microscope images of the grating couplers. (c, d) Photoluminance and Raman spectra of monolayer $\mathrm{WS}_{2}$ on the dielectric PC.

\section{Our sample was elaborately characterized at last. Figure S6a shows the bright-field optical} image of one sample studied in the main text. The distance between the gratings is $20 \mu \mathrm{m}$, which is the typical size for the exfoliated monolayer $\mathrm{WS}_{2}$. The grating parameters were confirmed by the atomic force microscope image as shown in Fig. S6b. The period of gratings is about $690 \mathrm{~nm}$. The width and depth of the grooves are $370 \mathrm{~nm}$ and $90 \mathrm{~nm}$, which were designed for the efficient excitation of BSWs for the FW laser.

$$
\text { After the fabrication of the grating couplers, a suitable } \mathrm{WS}_{2} \text { was transferred upon the }
$$
unstructured substrate. The photoluminescence and Raman spectra were measured to confirm the material quality. Figure S6c shows the photoluminescence spectrum excited by a $532 \mathrm{~nm}$ continuous wave laser. The strong photoluminescence peak is attributed to the optical transition of A exciton and the shoulder originates from the trion and defects emission $^{2}$. In the Raman spectrum shown in Fig. S6d, two prominent peaks appeared at $350 \mathrm{~cm}^{-1}$ and $420 \mathrm{~cm}^{-1}$, which are identified as the $2 \mathrm{LA}+\mathrm{E}^{\prime}$ and $\mathrm{A}_{1}{ }^{\prime}$ Raman modes, respectively. Those apparent spectroscopic features indicate the non-destructive quality of the transferred $\mathrm{WS}_{2}$. 

vector of BSWs

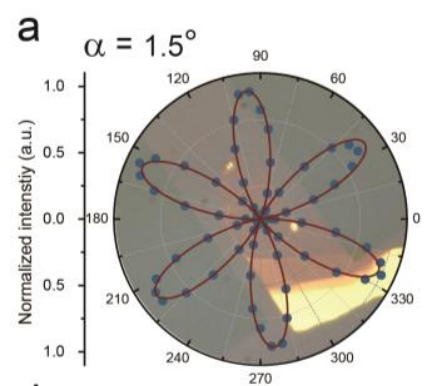

d

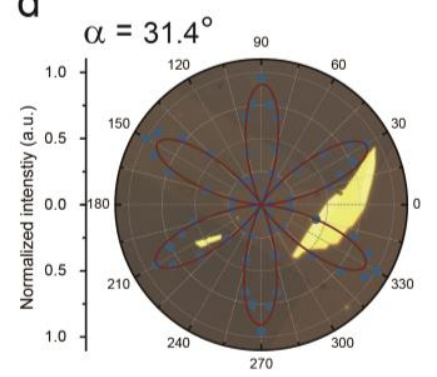

g $\alpha=20.7^{\circ} \quad 90 \quad$ SHG

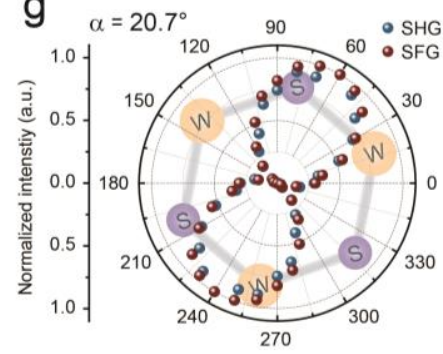

b

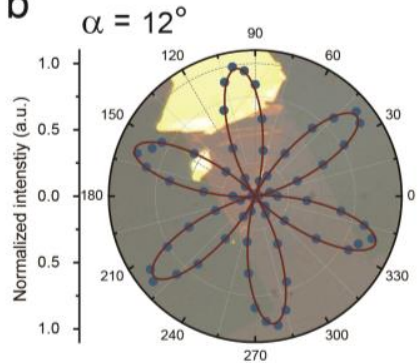

e

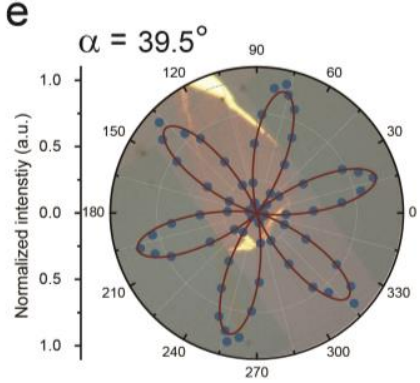

$\mathrm{h}$

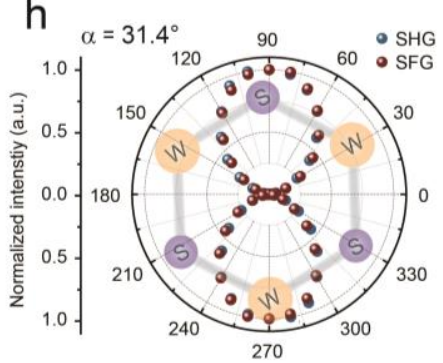

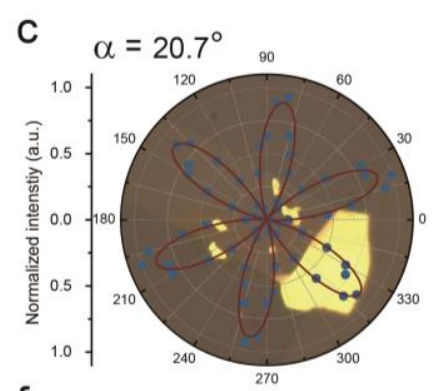

f
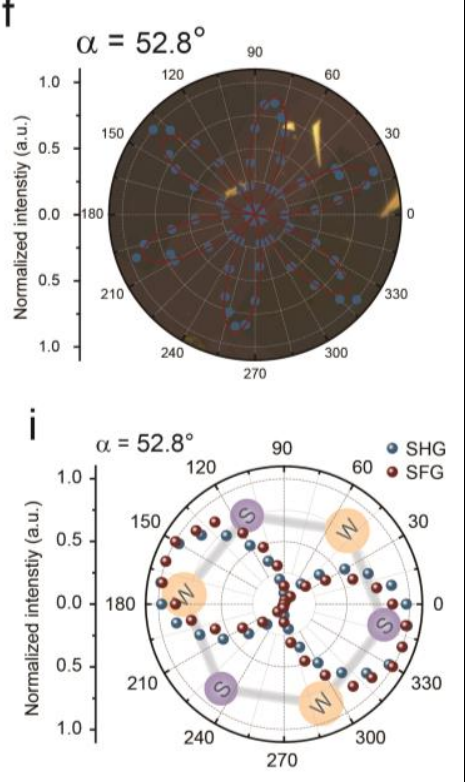

Figure S7. (a-f) Polarization-resolved SHG measurement for determining the crystal orientation of $\mathrm{WS}_{2}$. (g-i) SHG and SFG polarization distributions for the $\mathrm{WS}_{2}$ deposited at different twist angles.

To determine the twist angle between the $\mathrm{WS}_{2}$ and the wave vector of BSWs, polarization-resolved SHG was measured firstly to identify the crystal orientation of $\mathrm{WS}_{2}$. The samples were mounted on a rotatable stage and a picosecond pulsed laser at $800 \mathrm{~nm}$ (77 MHz, NKT Photonics, EXB-4) was used as an excitation source. The polarizations were in a co-linear configuration in the excitation and collection paths.

177 Rotating the sample and the SHG peaks can be used to identify the orientation axis of $178 \mathrm{WS}_{2}$. Several samples with different twist angles were prepared and the optical images were aligned with the polarized SHG as shown in Fig. S7a-f. By precise measurements, the angles of between the armchair axis and the wave vector of BSWs were obtained as labeled in corresponding figures. At the twist angles $\alpha=20.7^{\circ}, 31.4^{\circ}$, and $52.8^{\circ}$, the polarization distribution of SHG and SFG were measured (Fig. S7g-i). 
The SHG and SFG have the same polarization distribution and the results well agree with the theoretical prediction in the main text.

\section{S7 Estimating the conversion efficiency of SHG}

The nonlinear optical conversion efficiency was estimated by measuring all the related optical elements in our experimental setup. Firstly, the $800 \mathrm{~nm}$ femtosecond laser was guided through the same path as the SHG experiment. The FW power $\left(P_{\text {laser }}\right)$ was measured under the objective using a power meter. The coupling efficiency of the grating coupler was simulated with the same parameters in the experiment. The coupling efficiency for the laser to TE-BSWs is about $\eta_{\mathrm{gra}}=4 \%$ according to the simulations. The propagation loss can be neglected at such short distance considering the several-millimeters propagation length of BSWs. So the averaged power impinging on the $\mathrm{WS}_{2}$ is $\bar{P}_{\mathrm{FW}}=2 \cdot P_{\text {laser }} \cdot \eta_{\text {gra }}$ and the peak power is $P_{\mathrm{FW}}=\bar{P}_{\mathrm{FW}} /(v \tau)$, where $v$ and $\tau$ are the repetition rate and pulse duration, respectively. For the calibration of the collection efficiency for the SHG, a laser at $400 \mathrm{~nm}$ was guided through the same path except that the dichroic mirror (FF670-SDi01, Semrock) was replaced by a beam splitter $(R / T=3 / 7$ in the visible region). The transmittance ratio of every optical element was measured in the collection path, including the objective, dichroic mirror, and band-pass filter as listed in Table 1. To avoid the saturation of the CCD, a neutral density filter (transmittance 0.0249 at SH wavelength) was inserted before the CCD. The CCD detection efficiency was 0.4 provided by the factory. So the averaged SHG emission power $\left(\bar{P}_{\mathrm{SH}}\right)$ can be deduced from the above parameters.

The averaged SHG conversion efficiency $\left(\eta_{\mathrm{ave}}\right)$ can be denoted by $\eta_{\mathrm{ave}}=\bar{P}_{\mathrm{SH}} / \bar{P}_{\mathrm{FW}}^{2}$. The corresponding peak conversion efficiency of SHG was calculated by $\eta_{\text {peak }}=P_{\mathrm{SH}} / P_{\mathrm{FW}}^{2}$. Using this method, the averaged conversion efficiency of SHG for monolayer $\mathrm{WS}_{2}$ on the silica was estimated to be about $2.59 \times 10^{-8} / \mathrm{W}$, which is comparable with the previous reports ${ }^{3}$. The averaged (peak) conversion efficiency of SHG excited by BSWs was $8.26 \times 10^{-5} / \mathrm{W}\left(2.38 \times 10^{-10} / \mathrm{W}\right)$, more than three orders of magnitude larger compared with that in bare $\mathrm{WS}_{2}$. 
213 Table S1 Measured transmission ratio or detection efficiency of the optical elements 214 at the SH wavelength.

\begin{tabular}{cccccccc}
\hline & Objective & Dichroic mirror & Beamsplitter & Filter & ND filter & Lens & CCD \\
\hline \hline$T$ & 0.6856 & 0.9673 & 0.6291 & 0.9511 & 0.0249 & 0.9 & - \\
$\eta$ & - & - & - & - & - & - & 0.4 \\
\hline
\end{tabular}

\section{S8 The present conversion efficiency in comparison with the state of the arts}

217 To improve the nonlinear conversion efficiency in the layered TMDs, there are 218 mainly two scenarios in the previous reports. First, one can enhance the ambient 219 electromagnetic field response at FW and/or SHG frequency to improve the 220 nonlinearity. Second, the limited interaction length can be significantly prolonged by 221 coupling the guided evanescent with TMDs, resulting in larger nonlinear conversion 222 efficiency. In our experiment, the lossless BSWs have an optimized spatial overlap 223 with $\mathrm{WS}_{2}$, which is conducive to increasing the nonlinear interaction strength. More 224 importantly, the counter-propagating excitation method can break the limitation of the 225 phase-matching condition, which is usually satisfied by elaborately designing the 226 structure in other waveguides. The enhancement of the conversion efficiency or SHG 227 intensity for TMDs was summarized in Table S2. The present scenario in this work 228 possesses more extensibility and larger enhancement of the conversion efficiency. 
230 Table S2 SHG enhancement comparisons of the layered TMDs in different 231 configurations.

\begin{tabular}{|c|c|c|c|}
\hline $\begin{array}{l}\text { Enhanceme } \\
\text { nt schemes }\end{array}$ & Structure & Enhancement factor & References \\
\hline \multirow{9}{*}{$\begin{array}{c}\text { Field } \\
\text { enhancement } \\
\text { at the FW } \\
\text { and/or SH } \\
\text { frequency }\end{array}$} & $\begin{array}{c}\mathrm{WS}_{2}-\mathrm{SiO}_{2} \\
\text { microsphere }\end{array}$ & $20 \times$ enhancement & $\begin{array}{l}\text { Adv. Opt. Mater., } \\
\mathbf{2 0 1 8}, 7 \text { (3), } 1801270\end{array}$ \\
\hline & $\begin{array}{l}\mathrm{WSe}_{2}-\mathrm{DBR} \\
\text { structure }\end{array}$ & $58 \times$ enhancement & $\begin{array}{l}\text { ACS Appl. Mater. } \\
\text { Interfaces., 2018, 10, } \\
\text { 16874-16880 }\end{array}$ \\
\hline & $\begin{array}{l}\mathrm{MoS}_{2} \text {-Microca } \\
\text { vity }\end{array}$ & $10 \times$ enhancement & $\begin{array}{l}\text { Opt. Mater. Express, } \\
\text { 2016, } 6 \text { (7), } 2360\end{array}$ \\
\hline & $\begin{array}{c}\mathrm{MoS}_{2}-\text { Optical } \\
\text { cavity }\end{array}$ & $\begin{array}{c}5000 \times \text { enhancement } \\
\text { (peak conversion } \\
\text { efficiency } 1.62 \times 10^{-8} / \mathrm{W} \text { ) }\end{array}$ & $\begin{array}{l}\text { Nano Lett., 2016, } 16 \\
\text { (3), 1631-1636 }\end{array}$ \\
\hline & $\begin{array}{l}\text { WSe } 2 \text {-Photonic } \\
\text { crystal }\end{array}$ & $200 \times$ enhancement & $\begin{array}{l}\text { 2D Mater., 2016, } \\
4(1), 015031\end{array}$ \\
\hline & $\begin{array}{l}\mathrm{WSe}_{2} \text {-Gold } \\
\text { trenches }\end{array}$ & $7000 \times$ enhancement & $\begin{array}{l}\text { ACS Nano, 2018, } 12 \\
\text { (2), 1859-1867 }\end{array}$ \\
\hline & $\begin{array}{l}\mathrm{WS}_{2} \text {-Silver } \\
\text { nanogroove }\end{array}$ & $\begin{array}{l}400 \times \text { enhancement } \\
\left(2 \times 10^{-5} / \mathrm{W}\right)\end{array}$ & $\begin{array}{l}\text { Laser \& Photonics } \\
\text { Rev., 2018, } 12 \text { (10), } \\
1800188\end{array}$ \\
\hline & $\begin{array}{c}\mathrm{WS}_{2}-\mathrm{BIC} \\
\text { dielectric } \\
\text { metasurface }\end{array}$ & $1140 \times$ enhancement & $\begin{array}{l}\text { Nano Lett., 2020, 20, } \\
\text { 5309-5314 }\end{array}$ \\
\hline & $\begin{array}{c}\mathrm{WS}_{2} \text {-Plasmonic } \\
\text { nanocavity }\end{array}$ & $280 \times$ enhancement & $\begin{array}{l}\text { ACS Photonics, } \\
\mathbf{2 0 2 0}, 7(3), 562-568\end{array}$ \\
\hline \multirow{3}{*}{$\begin{array}{l}\text { Enhancing } \\
\text { interaction } \\
\text { length }\end{array}$} & $\begin{array}{l}\mathrm{MoSe}_{2} \text {-Silicon } \\
\text { waveguide }\end{array}$ & $280 \times$ enhancement & $\begin{array}{l}\text { Light: Sci. Appl., } \\
\text { 2017, } 6 \text { (10), e17060 }\end{array}$ \\
\hline & $\begin{array}{l}\mathrm{WS}_{2} \text {-Fiber } \\
\text { waveguide }\end{array}$ & $20 \times$ enhancement & $\begin{array}{l}\text { Light: Sci. Appl., } \\
\text { 2019, } 8,8\end{array}$ \\
\hline & $\mathbf{W S}_{2}$-BSWs & $\begin{array}{c}\text { 1000x enhancement } \\
\left(8.26 \times 10^{-5} / \mathrm{W}\right)\end{array}$ & This work \\
\hline
\end{tabular}


234

235

236

237

238

239

240

241

242

243

244

245

246

247

248

249

250

251

252

253

254

255

256

257

258

259 (4) Schuller, J. A.; Karaveli, S.; Schiros, T.; He, K.; Yang, S.; Kymissis, I.; Shan, J.;
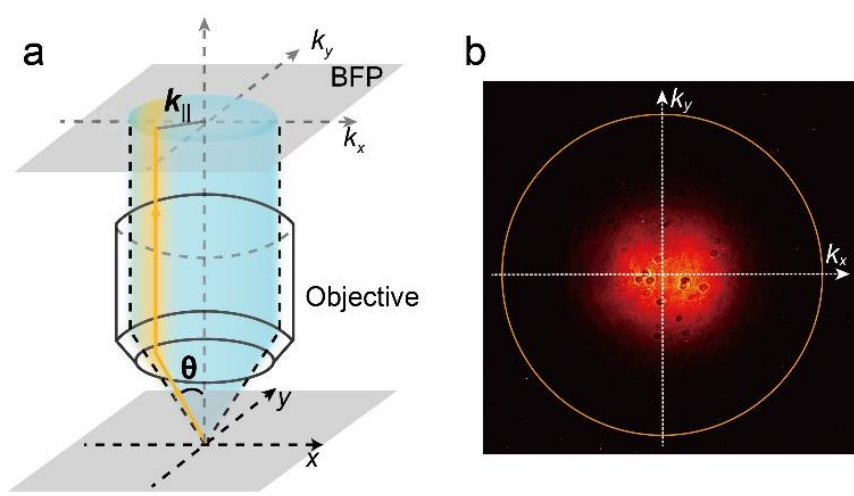

Figure S8. (a) Schematic of the back focal plane imaging microscopy. (b) In-situ SHG Fourier image obtained by the $50 \times$ objective with $\mathrm{NA}=0.5$.

In the atomically thick material, the requirement of phase matching was relaxed in the second-order nonlinear process. The SHG radiation can be considered as a series of in-plane nonlinear dipoles, which can be corroborated by the back-focal plane imaging of SHG. This technique has been utilized for quantifying the wave vector distribution of light or characterizing the radiative dipole $e^{4,5}$. The schematic diagram of the back focal plane imaging is shown in Fig. S8a. The emitted photon with an angle $\theta$ was collected by an objective, whose back focal plane was projected on a CCD for imaging. The Fourier image of in-situ excited SHG is shown in Fig. S8b. The radiation of SHG shows a homogeneous intensity distribution centered at the surface normal of $\mathrm{WS}_{2}$, which is consistent with the second-order nonlinear polarization of $\mathrm{WS}_{2}$.

\section{Reference}

(1) Chen, Y.-C.; Yeh, H.; Lee, C.-J.; Chang, W.-H., Distributed Bragg reflectors as broadband and large-area platforms for light-coupling enhancement in $2 \mathrm{D}$ transition-metal dichalcogenides. ACS Appl. Mater. Inter. 2018, 10 (19), 16874-16880. (2) Plechinger, G.; Nagler, P.; Kraus, J.; Paradiso, N.; Strunk, C.; Schüller, C.; Korn, T., Identification of excitons, trions and biexcitons in single-layer $\mathrm{WS}_{2}$. Phys. status solidi - RRL 2015, 9 (8), 457-461.

(3) Liu, X.; Zhang, Q.; Chong, W. K.; Yip, J. N.; Wen, X.; Li, Z.; Wei, F.; Yu, G.;

257 Xiong, Q.; Sum, T. C., Cooperative enhancement of second-harmonic generation from 58 a single CdS nanobelt-hybrid plasmonic structure. ACS Nano 2015, 9 (5), 5018-5026. 
260 Zia, R., Orientation of luminescent excitons in layered nanomaterials. Nat. 261 Nanotechnol. 2013, 8 (4), 271.

262 (5) Shi, J.; Li, Y.; Kang, M.; He, X.; Halas, N. J.; Nordlander, P.; Zhang, S.; Xu, H., 263 Efficient second harmonic generation in a hybrid plasmonic waveguide by mode 264 interactions. Nano Lett. 2019, 19 (6), 3838-3845.

265 